\title{
A New NS2 Tool to Investigate QoS Management over Mobile WiMAX
}

\author{
Alessandro Andreadis \\ University of Siena \\ Via Roma 56 \\ 53100 Siena, Italy \\ +3905771912432 \\ andreadis@unisi.it
}

\author{
Sandro Rizzuto \\ University of Siena \\ Via Roma 56 \\ 53100 Siena, Italy \\ +390577233601 \\ rizzuto@unisi.it
}

\author{
Riccardo Zambon \\ University of Siena \\ Via Roma 56 \\ 53100 Siena, Italy \\ +390577233601 \\ zambon@unisi.it
}

\begin{abstract}
WiMAX is one of the most interesting solutions for broadband wireless access, supporting multimedia traffics and applications through an appropriate QoS flow management both for fixed and mobile devices. To study its performance and to test new functionalities, simulation tools play a central role. In this paper we propose a new simulation module compliant to IEEE 802.16. Our key contribution is to offer the research community a useful tool for the investigation of QoS management on mobility in the WiMAX scenario. The developed tool includes the implementation of five QoS classes and of handover procedures. This module is validated and tested through exhaustive simulations, showing its conformance with the defined specifications.
\end{abstract}

\section{Categories and Subject Descriptors}

I.6.5 [Simulation and Modeling]: Model Development modeling methodologies

\section{General Terms}

Algorithms, design, performance, verification.

\section{Keywords}

WiMAX, mobility, QoS, scheduling, simulation.

\section{INTRODUCTION}

The IEEE 802.16 standard [1], known as WiMAX (Worldwide Interoperability for Microwave Access), is one of the most attractive solutions developed in the last years for the wide adoption of broadband wireless access to metropolitan areas. This standard specifies the physical and the MAC layers of the ISOOSI stack. It also provides Quality of Service (QoS) management Permission to make digital or hard copies of all or part of this work for personal or classroom use is granted without fee provided that copies are not made or distributed for profit or commercial advantage and that copies bear this notice and the full citation on the first page. To copy otherwise, to republish, to post on servers or to redistribute to lists, requires prior specific permission and/or a fee.

SIMUTOOLS 2011, March 21-25, Barcelona, Spain

Copyright (c) 2011 ICST 978-1-936968-00-8

DOI 10.4108/icst.simutools.2011.245513 through the adoption of five types of service flows: Unsolicited Grant Service (UGS), real-time Polling Service (rtPS), extended real-time Polling Service (ertPS), non real-time Polling Service (nrtPS) and Best Effort (BE). Moreover, this architecture supports mobility through the definition of scanning functionalities, three types of hand-over procedures, sleep mode for battery saving, etc.

Simulations can be a powerful tool to evaluate and enhance performance of WiMAX networks and to test the related functionalities. They provide the possibility to quickly set up different scenarios and to test wider and more complex solutions than in laboratory testbeds. Among the tools available in the research community for network simulation, the Network Simulator 2 (NS2) [2] is one of the most diffused and valuable solutions; it is a public domain tool implementing several protocols for wireless and wired architectures.

During the past years, several WiMAX modules were proposed for simulating IEEE 802.16-based networks. One of them, provided by National Institute of Standards and Technology [3], implements Wireless MAN-OFDM physical layer, adopting Time Division Duplexing (TDD) mode. It also implements mobility and includes enhancements in support of vertical handover. However, as regards QoS management, it is not fully compliant to the standard. In order to fill this gap, an additional module has been introduced in [4], implementing QoS classes, their requirements and specific signaling as defined by the standard. This module also includes some scheduling mechanisms, but it only implements UGS, rtPS and BE classes, without mobility support. The solution described in [5] implements the five WiMAX QoS classes and it is designed for TDD mode, but it does not support mobility and handover procedures defined by the IEEE 802.16 standard.

At the time of writing, the research community does not offer an NS2 module providing both WiMAX-compliant mobility support and QoS management, hence it is not possible to evaluate and to develop innovative solutions, aiming to preserve or enhance QoS management during mobility through different WiMAX networks. For this reason, in this paper we describe the development of a new simulation module for NS2, designed to be compliant with IEEE 802.16 standard. This module supports QoS management during mobility in WiMAX scenarios, offering the possibility for developers to work on this interesting research area. The module has been validated and tested through extensive simulations. 
This paper is organized as follows: in section 2 a brief overview of WiMAX and related standards is provided, focusing on QoS management and describing how IEEE 802.16 faces the mobility issue. Section 3 provides a detailed description of the module architecture and of the implemented IEEE 802.16 characteristics, concerning intra-inter cell mobility and QoS aspects in a Mobile WiMAX environment. Section 4 is dedicated to the validation of the module through extensive simulations and section 5 presents the concluding remarks.

\section{WIMAX OVERVIEW}

The Broadband Wireless Access technology named WiMAX is based on 802.16 standard [1]. It provides large coverage and high throughput, reaching theoretically a coverage radius up to 40 kilometers with a throughput of $75 \mathrm{Mb} / \mathrm{s}$ [6]. In practice, it is observed that WiMAX covers an area of about $20 \mathrm{~km}$ radius reaching $9 \mathrm{Mb} / \mathrm{s}$ using UDP and $5 \mathrm{Mb} / \mathrm{s}$ using TCP [7].

The main elements of WiMAX architecture are:

- Base Station (BS): the gateway between the wired and the wireless part of the system, its antenna covers a radius of several kilometers;

- Subscriber Station (SS): in the basic topology, it communicates with other SSs via the BS in a star connection mode; it provides connectivity to the subscriber equipment (e.g., notebook, PDA, etc.), it could also be indicated as Mobile Station (MS).

BS and SSs can be connected in two different modes, namely Point-to-Multipoint (PMP) and Mesh mode. In PMP mode, data are exchanged between BS and all SSs in broadcast way. BS centrally coordinates all data traffics, establishing for each SS a single uplink channel for its data and transmission requests. In mesh mode, traffic is managed and exchanged directly between SSs as well as with a BS; there are two alternatives: centralized (BS manages requests and bandwidth grants for SSs) and distributed (SSs can communicate among them with or without preventive agreement).

This work focuses on PMP mode, where BS provides full-duplex communication with SS or another BS, granting channel resources for WiMAX connections.

The PHY Layer supports Time Division Duplexing (TDD) access, at frequencies ranging from 2 to $11 \mathrm{GHz}$ with Orthogonal Frequency Division Multiplexing (OFDM). Frequency Division Duplexing (FDD) can be used as well.

The IEEE 802.16 standard supports four Modulation Coding Schemes (MCS): BPSK (Binary Phase Shift Keying), QPSK (Quadrature Phase Shift Keying), 16-QAM (Quadrature Amplitude Modulation) and, optionally, 64-QAM. The BS compares the Signal-to-Noise Ratio (SNR) value of the SS with suitable thresholds, in order to select the most appropriate MCS and consequently the highest available bit-rate for that connection.

The MAC frame is divided into two sub-frames for data transmission in downlink and uplink directions.
To support a wide variety of multimedia applications, the IEEE 802.16 standard defines five classes of service flows, each with different QoS requirements [4]:

- Unsolicited Grant Service (UGS): designed to support real-time traffics, very sensitive to transmission delay; it is characterized by fixed-size data packets, requiring fixed bandwidth allocation. BS provides grants in unsolicited manner;

- Real-Time Polling Service (rtPS): similarly to UGS, it deals with real-time traffics, but more delay-tolerant data are considered, with variable packet size. For this service flow class, BS periodically executes an individual polling for SSs bandwidth requests;

- Extended Real-Time Polling Service (ertPS): this class, introduced later, supports real-time traffics and represents an intermediate class between UGS and rtPS. The BS provides grants in unsolicited manner (as in UGS) with dynamic bandwidth allocation, and it is designed for variable rate sources;

- Non Real-Time Polling Service (nrtPS): it is associated with non real-time traffics; it could require a minimum reserved bit-rate. Again, the BS performs individual polling for SSs bandwidth requests;

- Best Effort (BE): it is designed for applications without guarantees in terms of delay or bit-rate. SSs send their bandwidth requests through a channel contention mechanism.

Each connection between SSs and BS is associated to one service flow, and there are three types of messages for service flow management:

- Dynamic Service Addition (DSA) for adding a new service flow;

- Dynamic Service Change (DSC) for modifying service flow parameters;

- Dynamic Service Delete (DSD) for deleting an existing service flow.

In WiMAX PMP architecture, three scheduling sessions can be envisaged [1]:

- SS uplink scheduling, to manage outbound flows towards the BS;

- BS downlink scheduling, to manage outbound flows towards each SS;

- BS request/grant uplink scheduling, performed by the BS to provide each subordinate SS with bandwidth for uplink transmissions, or opportunities to request resources.

IEEE 802.16 MAC layer defines QoS classes and their parameters, but the scheduling algorithms to be adopted in order to satisfy QoS requirements remain an open issue.

Concerning mobility, WiMAX supports seamless handoff to enable the MS to switch from one base station to another at vehicular speeds $(120 \mathrm{Km} / \mathrm{h})$, in order to ensure real-time 
applications without service degradation [8]. The 802.16 standard envisages three handoff methods [1]:

- Hard Handoff (HHO): MS switches from one BS to another;

- Fast Base Station Switching (FBSS): MS receives/transmits data from/to the Anchor BS that may change within the Diversity Set;

- Macro Diversity Handover (MDHO): MS transmits to and receives from multiple BSs at the same time.

The first method (HHO) is mandatory, while FBSS and MDHO are two optional modes. Moreover, several techniques were developed in order to optimize hard handoff, with the aim to keep layer 2 handoff delays below 50 milliseconds.

Finally, Mobile WiMAX supports a 3-way handshake scheme to optimize the re-authentication mechanisms for supporting fast handovers.

\section{WIMAX SIMULATION MODULE}

The proposed module is developed for the NS2 simulator release 2.29. It is designed to be compliant with IEEE 802.16 standard, in order to provide QoS management on mobility in WiMAX scenarios, on PMP network topology and TDD mode. To be easily integrated in the NS2 framework, it is implemented in the object oriented $\mathrm{C}++$ code.

The module design is based on the popular WiMAX tool described in [3]; it implements OFDM PHY with the adoption of the four MCS defined by the standard and it allows to configure parameters such as cyclic prefix, transmission power, frequency bandwidth, sampling frequency, OFDM symbol time duration, packet transmission time.

Concerning the MAC layer, some management messages such as downlink/uplink channel descriptors, downlink/uplink MAP, ranging request/response, registration request/response are implemented.

Figure 1 shows the MAC diagram representing the main class, Mac802_16, and its relations with other classes.

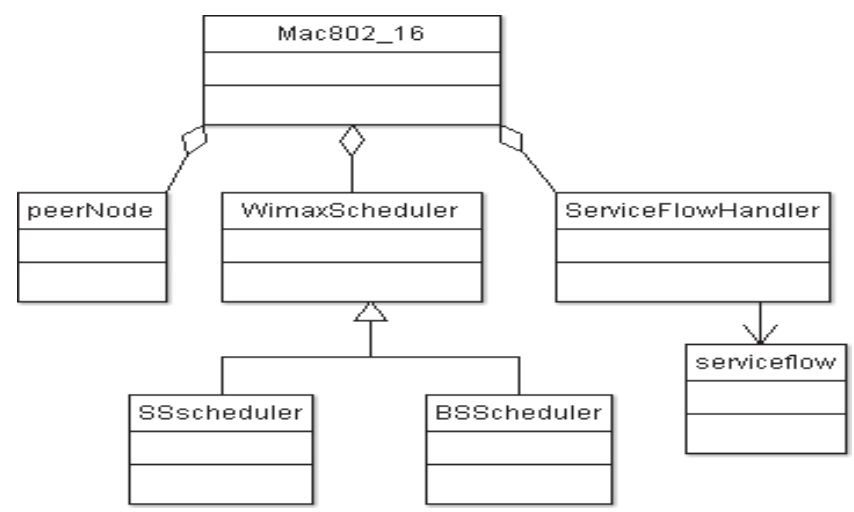

Figure 1. 802.16 MAC class diagram.

ServiceFlowHandler manages downlink/uplink connections and it associates to each connection a service flow with the required
QoS parameters; peerNode notifies the BS about channel parameters (SNR, MCS, etc.) of the associated SS; WimaxScheduler contains two schedulers, one for the BS (BSScheduler) and one for the SS (SSscheduler), to manage registration and allocation of network resources of the SSs.

The existing module supports mobility too. Specifically, the MS may perform scanning and handover operations between BSs.

As regards the new proposed module, code reuse was possible in the implementation of the described functionalities. However, several modifications and important integrations to the existing module were necessary in order to provide a simulation tool strictly compliant to IEEE 802.16 standard for QoS management during mobility.

In the following subsections our contribution is described in details.

\subsection{Link Adaptation}

The NIST module provides BPSK, QPSK, 16QAM and 64QAM MCSs. However, in this implementation the MCS is previously chosen for all SSs and does not change during the simulation.

Our contribution, similarly to [4], regards the implementation of link adaptation functionalities. MCS switches depending on the SNR values at the receiver, as defined in the standard (see Table 1 ), in order to guarantee a good link quality.

Table 1. Receiver SNR assumptions

\begin{tabular}{|c|c|c|}
\hline Modulation & Coding rate & Receiver SNR (dB) \\
\hline BPSK & $1 / 2$ & 3.0 \\
\hline QPSK & $1 / 2$ & 6.0 \\
\hline QPSK & $3 / 4$ & 8.5 \\
\hline 16-QAM & $1 / 2$ & 11.5 \\
\hline 16-QAM & $3 / 4$ & 15.0 \\
\hline 64-QAM & $2 / 3$ & 19.0 \\
\hline 64-QAM & $3 / 4$ & 21.0 \\
\hline
\end{tabular}

To obtain link adaptation, some modifications are needed in the PeerNode and BSScheduler class, in order to determine the suitable MCS depending on SNR changes.

In [4], SNR levels at the receivers are generated during simulations in an arbitrary mode, by the random generation of SNR values within $3.0 \mathrm{~dB}$ and $21 \mathrm{~dB}$. Instead, in our implementation, a function is added in the MAC802 16 class, for retrieving SNR from the PHY layer. These SNR values are computed by considering the path loss introduced in the wireless channel. In the simulation scenario, the path-loss is strictly dependent on MS position (movement) and its distance from the $\mathrm{BS}$, so this modification provides more realistic simulations in case of intra and inter-cell mobility.

In fact, different locations assumed by different MSs (or by the same MS in different time intervals) lead to dissimilar SNR values. Link adaptation implemented in the module chooses the best MCS, changing the number of available OFDM symbols for 
each MS. In this way, at different positions correspond different resource availability for the mobile stations, with obvious consequences on QoS management and performance.

\subsection{Quality of Service}

In order to implement QoS capabilities, we have adopted the IEEE 802.16 MAC class structure shown in Figure 1.

Some modifications to DSA packets (exchanged during a new flow addition) have been made so as to manage QoS according to IEEE 802.16 standard. Specifically, when a new service flow is added, some QoS parameters are provided, such as Connection Identifier, Traffic Priority, Maximum Sustained Traffic Rate, Minimum Reserved Traffic Rate, Tolerated Jitter and Maximum Latency. The ServiceFlowHandler class manages these parameters for the creation of new uplink and downlink connections.

The resource allocation consists of downlink bandwidth (BS outbound scheduler) and a portion of uplink bandwidth assigned by the BS uplink scheduling (SS outbound scheduler). In the previous module defined in [4], QoS class mapping has been implemented for the BS request/grant uplink scheduler. This scheduler manages in priority manner only three different classes, serving first UGS, secondly rtPS, and finally BE connections. Within each priority class, the reserved OFDM symbols are distributed among the various traffics, depending on the adopted scheduling mechanism (Figure 2).

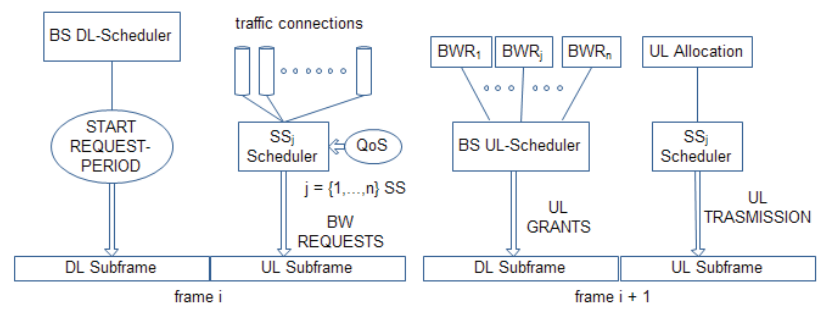

Figure 2. The different schedulers implemented at BS and MSs.

Our contribution to QoS class implementation consists in partial code reuse from [4], where only UGS, rtPS and BE classes were developed. UGS connections are served periodically with fixed bandwidth assignment determined by the Maximum Sustained Traffic Rate (MSTR) parameter. The available number of OFDM symbols reserved by the BS must be sufficient to serve all UGS connections.

As for rtPS connections, the BS determines the number of symbols to be allocated, basing on bandwidth requests presented by SSs during the polling period.

As for BE traffics, the BS reserves available bandwidth to SS traffics which have data to send and have won the contention request opportunity.

Concerning ertPS class, it works like UGS and rtPS. Transmission is performed during periodic time intervals allocated by the BS with dynamic size of grants, by modifying the MSTR value; this value is explicitly notified by the SS through polling opportunities or piggyback mechanisms.
Finally, the nrtPS class competes with other rtPS or BE traffics during BS scheduling period, in order to gain the demanded bandwidth.

With the complete implementation of the five QoS scheduling types, it is possible to map opportunely the different traffics in the simulated WiMAX network.

\subsection{Mobility Management}

The previous module supports mobility, implementing the mandatory $\mathrm{HHO}$ standard. Due to signal fading, interference levels, and other channel conditions related to mobility, the MS might need to execute a handover.

The handover procedure, including exchanged messages implemented in the module, is shown in Figure 3:

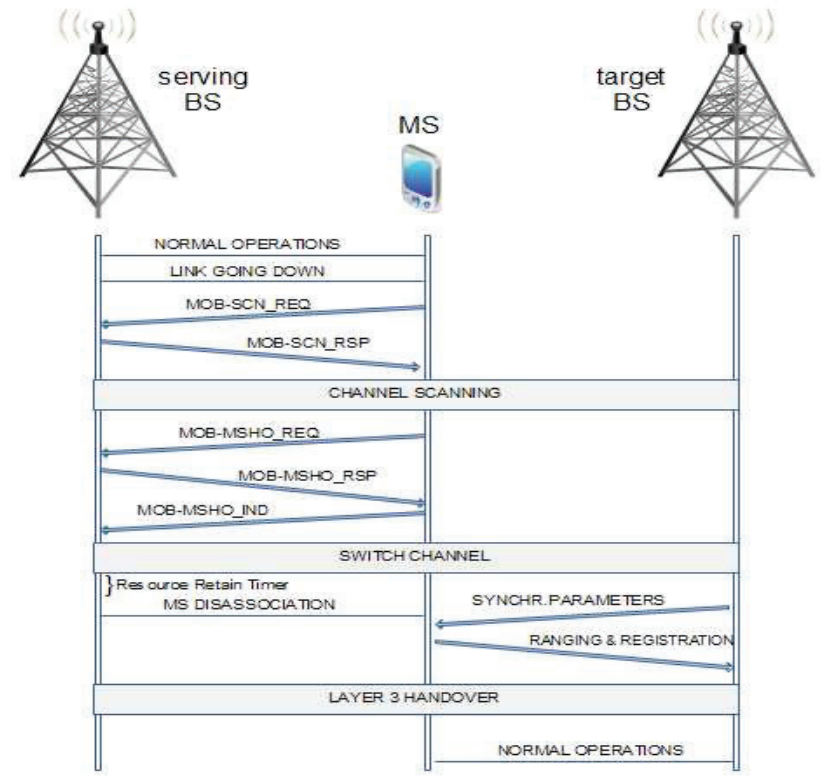

Figure 3. Handover procedure.

- the MS monitors the link status; if the link quality deteriorates, the MS sends a Scanning Interval Allocation Request (MOB-SCN_REQ) to the serving $\mathrm{BS}$, at the aim to request a scanning interval for finding surrounding BSs (an MS may terminate scanning and return to Normal Operation anytime, by sending a MAC PDU to the BS).

- The serving BS responds with a Scanning Interval Allocation Response (MOB_SCN-RSP) message (the MS may retransmit the MOB_SCN-REQ message if it does not receive the MOB-SCN-RSP message within a pre-defined time).

- When a MS decides to pass from the serving BS to the target BS, it starts the handover through a Mobile Station Handover Request MOB_MSHO-REQ message (MOB_BSHO-REQ message is used when the handover is originated by the serving $\mathrm{BS}$ ). 
- MS obtains DL and UL transmission parameters in order to be synchronized to the target BS.

- Finally, MS leaves the serving BS, by sending a Handover Indication (MOB_HO-IND) message.

In [3], the handover procedure is concluded at this step. However, when the MS leaves a serving BS, no resource release procedure is implemented, with consequent bandwidth waste.

For this reason, in order to implement a complete handover procedure fully compliant to the IEEE 802.16 standard, we have introduced a Resource Retain timer. The serving BS will retain the connections, MAC state machine, and PDUs associated with the MS for service continuation until the expiration of resource retain timer. In fact, for different reasons, an MS may cancel the handover at any time before the expiration of the timer. On the other hand, if a handover is successfully accomplished, the moving MS can correctly exploit target BS network resources, and the previously serving BS can allocate new resources to other MSs.

The key contribution provided in the proposed module regards QoS management over mobility. An MS can cross several cells, by performing handover procedures needed to provide the correct resource allocation in the different cells. However, QoS parameters are already defined inside the MS and they are maintained through its inter-cell mobility.

In the NIST module, when an MS performs a handover from a serving BS to a target BS, the PeerNode class associated with the MS changes, so QoS parameters information are not transferred to the new class and the BSScheduler is not able to manage traffic flows opportunely. Our contribution is the following: when an MS is linked with the target BS, its QoS information and all the other parameters are transferred to a new PeerNode associated to the SS. In this way, the BSScheduler class receives bandwidth requests from traffics and maintains the association with corresponding QoS identifier, in order to activate the suitable uplink scheduler and to allocate network resources.

Finally, although layer 2 mobility is implemented as described, in NS2 simulation scenarios each BS is addressed on a different subnet (i.e. domain). Therefore a layer 3 handover is employed to provide seamless mobility through different BSs. After the execution of a handover, a Neighbor Discovery [3] module is adopted to provide layer 3 mobility detection functionalities. Then, a new NS layer 3 address is needed for the MS. Finally, when a new address is assigned to the agent node (MS), a BS route update is performed.

\subsection{Scheduling}

The developed tool includes several scheduling algorithms and provides the possibility to choose between Round Robin, Maximum Signal to Interference Ratio, Weighted Round Robin, and Temporary Removal schedulers [9].

Moreover, it adds the DRR scheduler [10] as an interesting solution for enhancing multi-rate fairness.

\section{SIMULATION RESULTS}

In this section we check the compliance of the proposed module to the IEEE 802.16 standard for PMP topology and TDD. In order to validate different functionalities, three different scenarios were envisaged:

- A fixed scenario, to demonstrate the link adaptation implementation and the relationship between the MS location and allocated resources;

- Another fixed scenario, to validate the IEEE 802.16 UGS, ertPS, rtPS, nrtPS and BE scheduling services and the standard compliant QoS slots allocation;

- A mobile WiMAX scenario, to validate mobility functionalities and support of QoS classes during MS movements through different BSs.

The main parameters used to simulate the WiMAX network are the same used in [3] and are reported in Table 2.

Table 2. Main parameters of the simulation model.

\begin{tabular}{|c|c|}
\hline Parameter & Value \\
\hline Frequency band & $5 \mathrm{MHz}$ \\
\hline Propagation model & Two Ray Ground \\
\hline Antenna model & Omni antenna \\
\hline Antenna height & $1.5 \mathrm{~m}$ \\
\hline Transmit antenna gain & 1 \\
\hline Receive antenna gain & 1 \\
\hline System loss factor & $15 \mathrm{~W}$ \\
\hline Transmit power (BS) & $500 \mathrm{~m}$ \\
\hline Coverage radius & $1,22 \mathrm{E}-009$ \\
\hline Receive power threshold & $9,72 \mathrm{E}-010$ \\
\hline Carrier sense power threshold & Enabled \\
\hline Link adaptation & 4 ms \\
\hline Frame duration & 0.25 \\
\hline Cyclic prefix (CP) & 1024 bytes \\
\hline Packet length & \\
\hline
\end{tabular}

\subsection{Link Adaptation Validation}

The first scenario validates the correct trend of the Signal to Noise Ratio on the basis of the MS position and, consequently, how it affects MCS selection and the related available bandwidth for the considered MS.

The simulation scenario (named SIM1) represents a WiMAX PMP infrastructure, composed by the BS, connected to a fixed host through a Gigabit Ethernet switch, and an MS (see Figure 4). The MS generates a Constant Bit Rate (CBR) UDP uplink traffic towards the fixed host. The bit-rate of the CBR is $5 \mathrm{Mb} / \mathrm{s}$ and it is mapped in the rtPS class. The MS moves from a position close to the BS, characterised by a high SNR value, and crosses the cell border (at about 60s simulation time). Then, the MS returns back to the initial position (at 100s), thus experiencing different channel conditions. CBR traffic is active for the whole duration of simulation (i.e., 100s). The main simulation parameters are those reported in Table 2. 


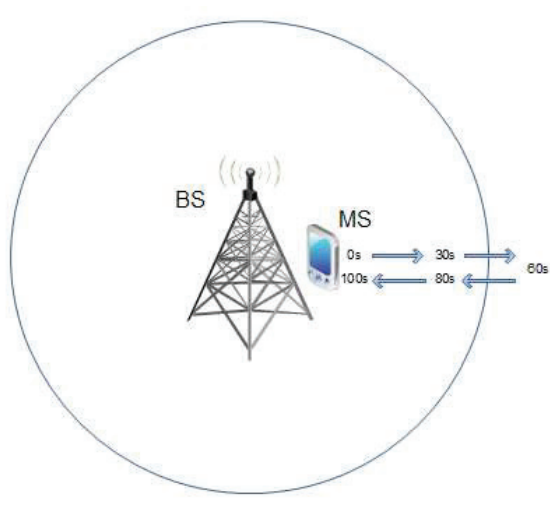

Figure 4. First simulation scenario (SIM1).

Table 3 reports, at different times, the SNR at the MS, the MCS selected by the link adaptation policy implemented in the module and, consequently, the CBR flow rate for the MS uplink data transmission.

Table 3. Resource assignment on mobility

\begin{tabular}{|c|c|c|c|}
\hline Time (s) & SNR (dB) & MCS & Rate $(\mathbf{M b} / \mathbf{s})$ \\
\hline 0 & 17,5 & 16QAM 3/4 & 5 \\
\hline 10 & 17,5 & 16QAM 3/4 & 4,99 \\
\hline 20 & 13,63 & 16QAM 1/2 & 3,19 \\
\hline 30 & 10,13 & QPSK 3/4 & 2,27 \\
\hline 40 & 6,82 & QPSK 1/2 & 1,51 \\
\hline 50 & 2,67 & BPSK 1/2 & 0,78 \\
\hline 60 & 0,84 & BPSK 1/2 & 0 \\
\hline 70 & 6 & QPSK 1/2 & 0 \\
\hline 80 & 6,27 & QPSK 1/2 & 1,55 \\
\hline 90 & 10,04 & QPSK 3/4 & 2,28 \\
\hline 100 & 13,5 & 16QAM 1/2 & 4,96 \\
\hline
\end{tabular}

The SNR values changes contextually with the MS position, influencing its transmission rate. Here, the implemented link adaptation switches to the most suitable MCS on the basis of values reported in Table 1, thus providing the best trade-off between link quality and robustness.

In Table 3 we can note that, at time $=60 \mathrm{~s}$, the SNR value is lower than BPSK threshold defined by the standard (see Table 1); for this reason, although the chosen MCS is BPSK, no data is transmitted (a similar behavior occurs at time $=70$ s with QPSK).

\subsection{QoS Management}

The second simulation scenario (named SIM2) is designed to check whether the resource allocation of data packets follows the rules specified for the five QoS service classes.
The topology represents a WiMAX PMP infrastructure, composed by the BS, connected to a fixed host through a Gigabit Ethernet switch, and a SS (see Figure 5).

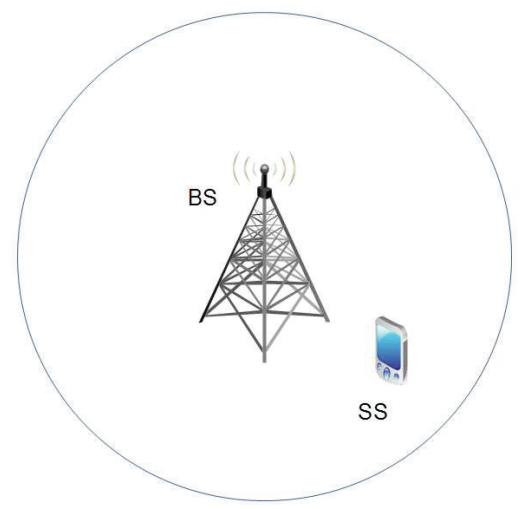

Figure 5. Second simulation scenario (SIM2).

During each simulation run, the SS is involved in different traffics:

- UGS validation (SIM2a): the SS generates uplink VoIP traffic, according to an exponential model with burst time of $1.2 \mathrm{~s}$, idle time of 1.8 and data packets of 66 bytes in length generated at fixed intervals of $20 \mathrm{~ms}$;

- $\quad$ ertPS validation (SIM2b): the SS generates an uplink VoIP traffic with silent suppression, modeled as above, with the exception that the traffic rates are randomly chosen among three different values: 171 bits/packet, 80 bits/packet and 40 bits/packet;

- $\quad$ rtPS validation (SIM2c): the SS is involved with the fixed host in an uplink streaming video session, generated by real MPEG traces [5];

- $\quad$ nrtPS validation (SIM2d): the SS is involved with the fixed host in a file transfer, modeled as an FTP traffic source with packet size of 1024 bytes;

- $\quad \mathrm{BE}$ validation (SIM2e): the $\mathrm{SS}$ is involved in a web browsing session, modeled as a hybrid Lognormal/Pareto distribution, with an area of 0.88 and mean of 7247 bytes for the Lognormal distribution and 10588 bytes for the Pareto distribution.

As before, the main simulation parameters are reported in Table 2.

Figure 6 reports the OFDM symbol allocation in the UGS case (SIM2a). In the X-axis the simulation time is reported, in the Yaxis the allocated symbols are shown. As described in Table 2, the frame duration is $4 \mathrm{~ms}$. In fact, the interval between two successive data transmission is equal to the frame duration. BS provides data grants to the UGS periodically, with a fixed allocation. It correctly fits a CBR flow. 


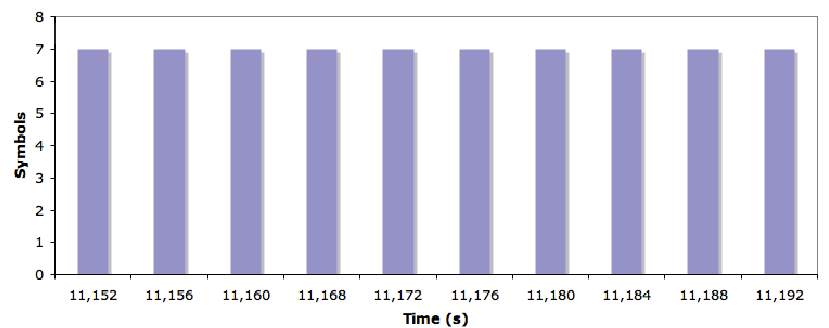

Figure 6. UGS validation (SIM2a).

In figure 7, resource allocation in terms of symbols is reported for the ertPS case (SIM2b). Even in this case, it is possible to see the grants. However, now allocation is dynamic, in order to better manage the burst generated by a bursty source such as VoIP with silent suppression.

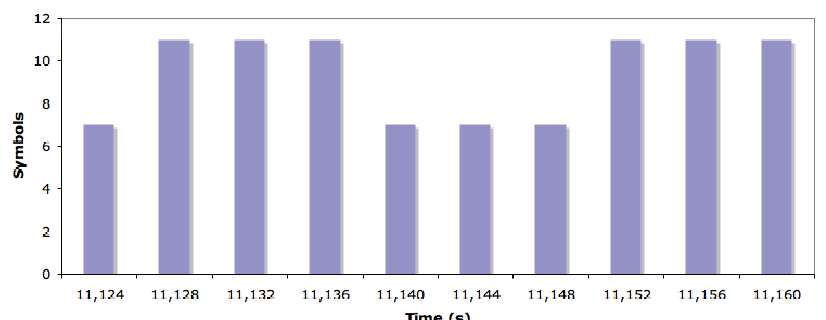

Figure 7. ertPS validation (SIM2b).

Figure 8 shows rtPS behavior (SIM2c). The polling service permits to reserve variable resources for the VBR connection.

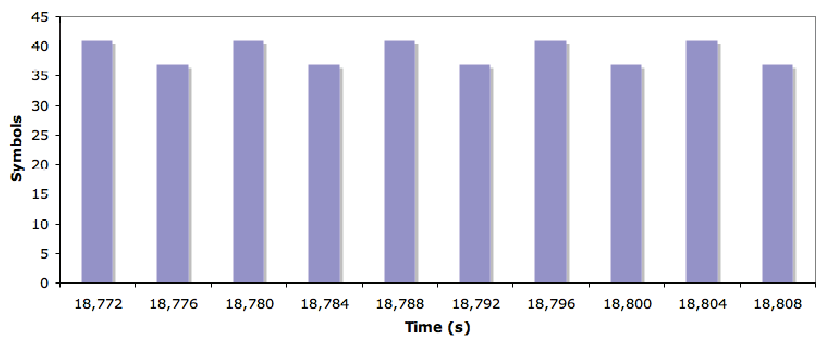

Figure 8. rtPS validation (SIM2c).

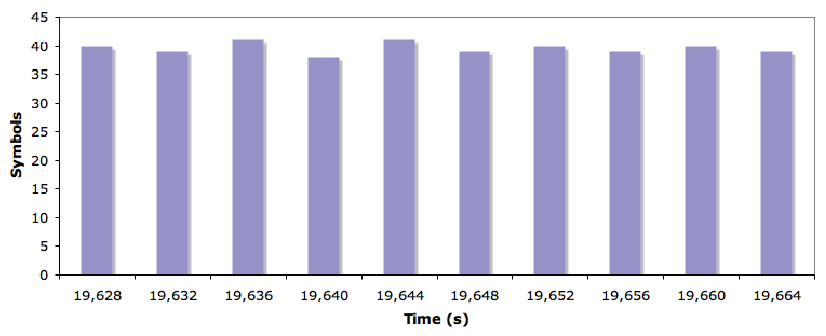

Figure 9. nrtPS validation (SIM2d).
Figure 9 reports nrtPS scenario (SIM2d), where the FTP traffic requires variable resources too.

Figure 10 represents the best effort case (SIM2e). In this case, no grants or resource reservation is provided through bandwidth requests.

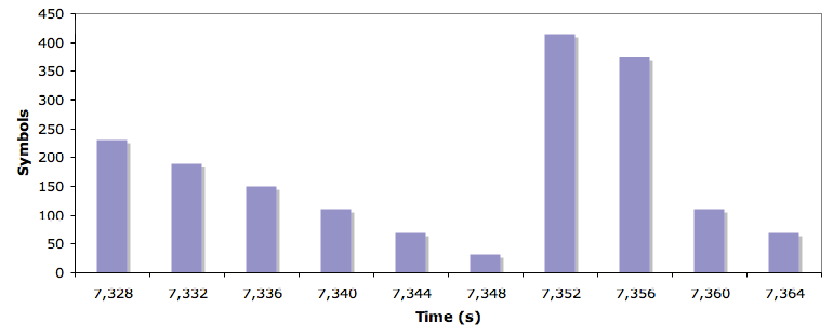

Figure 10. BE validation (SIM2e).

\subsection{QoS on Mobility}

In this section, the tool is validated with respect to QoS support during mobility; QoS classes prioritization is maintained during MS mobility through different WiMAX cells.

The topology adopted in this case represents a WiMAX PMP infrastructure, composed by three BSs serving three contiguous cells, connected to a fixed host through a Gigabit Ethernet switch. (see Figure 11).

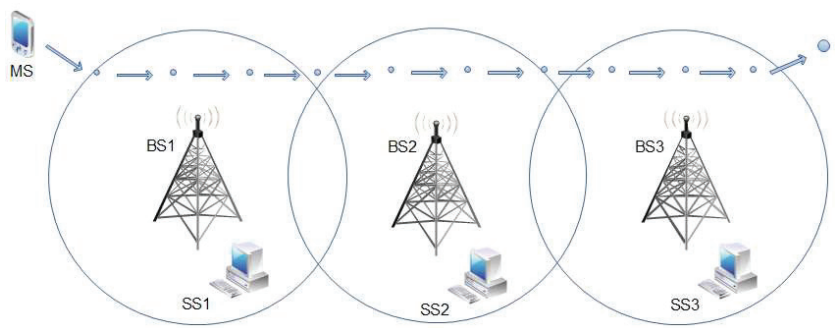

Figure 11. Third simulation scenario (SIM3).

An $\mathrm{SS}$ is active in each cell ( $\mathrm{SS} 1, \mathrm{SS} 2, \mathrm{SS} 3)$ and communicates with its serving BS (BS1, BS2, BS3 respectively) with the following traffic:

- SS1: CBR flow mapped as UGS, with rate at $120 \mathrm{~Kb} / \mathrm{s}$ and packet size of 1024 bytes;

- $\quad$ SS2: CBR flow mapped as rtPS, with rate at $1,7 \mathrm{Mb} / \mathrm{s}$ and packet size of 1024 bytes;

- $\quad$ SS3: CBR flow mapped as BE, with rate at $1,7 \mathrm{Mb} / \mathrm{s}$ and packet size of 1024 bytes;

Furthermore, a mobile station (MS) crosses the three cells, starting from cell1 (at 0 s of simulation time) and ending to cell 3 
(at the end of simulation time, 200s), performing WiMAXcompliant handovers.

The MS is involved in different traffics, always modeled as CBR flows with bit-rate $200 \mathrm{~Kb} / \mathrm{s}$ and packet size 1024 bytes. However, in each simulation run the CBR flow is mapped into a different QoS class, as follows:

- UGS validation on mobility (SIM3a): CBR flow mapped as UGS;

- $\quad$ ertPS validation on mobility (SIM3b): CBR flow mapped as ertPS;

- $\quad$ rtPS validation on mobility (SIM3c): CBR flow mapped as rtPS;

- $\quad$ nrtPS validation on mobility (SIM3d): CBR flow mapped as nrtPS;

- $\quad$ BE validation on mobility (SIM3e): CBR flow mapped as BE.

At this stage only CBR traffics are adopted, instead of more realistic sources for the five types of service flow. This solution is chosen to facilitate the analysis of results, as in [5], given the more complex scenario with respect to SIM1 and SIM2.

All traffics start at 0 s and end at 200s. Also in this case, the main simulation parameters are reported in Table 2.

In this particular scenario, the MS movement through different cells introduces handovers. In the time interval between $0 \mathrm{~s}$ and about $60 \mathrm{~s}, \mathrm{MS}$ is served by BS1. At about $60 \mathrm{~s} \mathrm{MS} \mathrm{performs} \mathrm{a}$ handover, switching its serving BS from BS1 to BS2 and entering cell2, where it remains till $130 \mathrm{~s}$. At this time, MS executes another handover and obtains association with BS3.

In the following figures, goodput performance related to the 4 WiMAX stations (SS1, SS2, SS3, MS) are reported. Specifically, in these simulations we validate the correct resource allocation for the five QoS classes and different resource access priorities.

Figure 12 shows the goodput performance for SIM3a, where MS traffic is mapped as UGS flow.

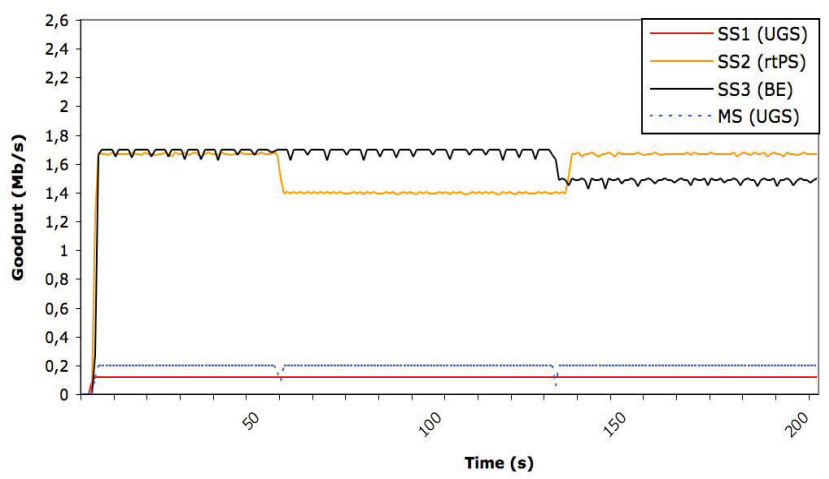

Figure 12. UGS goodput during mobility (SIM3a).
During MS movement within BS1 cell, MS and SS1 traffics are mapped as UGS, showing the same good performance. After the first MS handover, the MS traffic maintains its QoS characteristics and its resource exploitation is granted by BS2 with respect to SS2. In fact, the traffic generated by SS2 is managed as rtPS, showing performance degradation during the MS sojourn in cell2. After the second handover, MS maintains UGS granted resource allocation with good performance, at the expenses of SS3 traffic in cell3, mapped as BE. However, the goodput decrease of SS3 traffic is slightly lower than SS2 traffic, due to the fact that SS2 connection (rtPS) is managed through a polling mechanism, thus introducing more latency and reduced bandwidth efficiency with respect to $\mathrm{BE}$ connections. In conclusion, this scenario emphasizes the highest priority of UGS.

In Figure 13, MS traffic is mapped as ertPS (SIM3b). In this case, during MS sojourn within cell1, both traffic flows are correctly served due to the absence of congestion. However, when MS switches to BS2, the higher priority of ertPS keeps good performance to MS traffic, introducing performance degradation for SS2 rtPS flow. The same happens for SS3 traffic after the second handover of MS, being it mapped as a lowest priority flow.

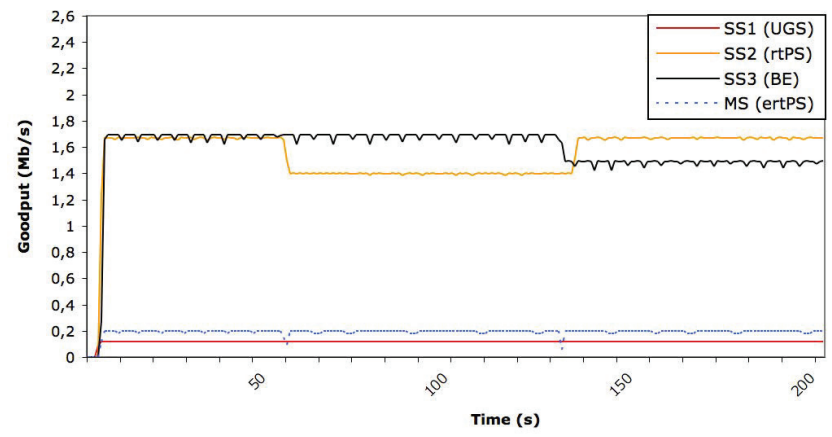

Figure 13. ertPS goodput during mobility (SIM3b).

In case of rtPS mapping for MS traffic flow (SIM3c, shown in Figure 14), in cell1 the resource allocation based on lower priority polling procedure introduces degradation only for MS traffic, while SS1 UGS flow maintains good performance due to its higher priority and its grant resource allocation mechanism. When MS performs handover to BS2, the available resources are equally shared between the two rtPS traffics (MS and SS2), with a similar QoS degradation. In the third phase of the simulation, MS exploits the needed resources from BS3, penalizing the SS3 BE traffic with lowest priority.

In SIM3d (Figure 15) the MS traffic mapped as nrtPS suffers between the first and the second handover. In fact, the resource assignment to MS is performed through polling and contention mechanisms, while SS2 rtPS flow resource allocation is provided through pure polling. However, this mixed assignment from BS3 provides resources to the nrtPS traffic, where SS3 BE traffic is affected by goodput degradation. 


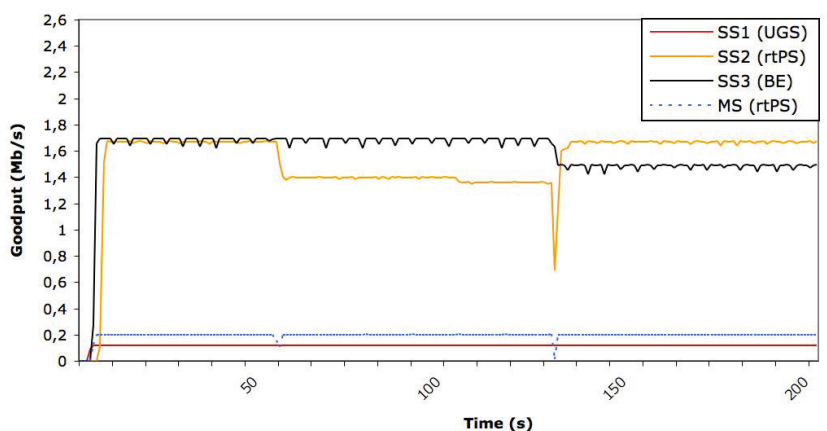

Figure 14. rtPS goodput during mobility (SIM3c).

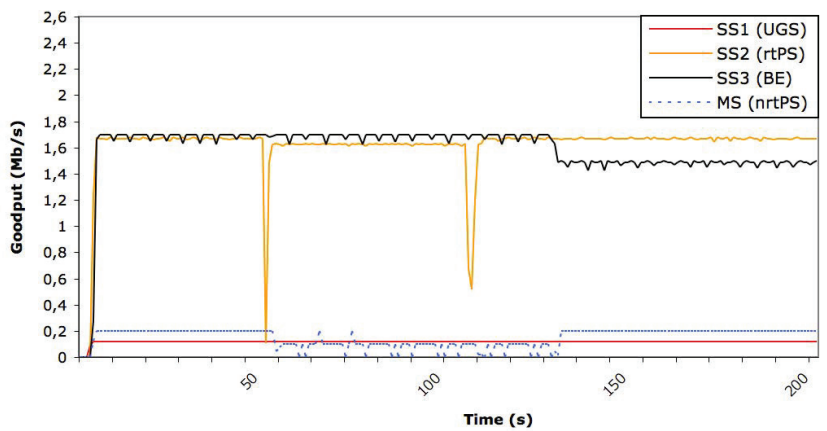

Figure 15. nrtPS goodput during mobility (SIM3d).

Finally, figure 16 reports the goodput performance for SIM3e, where the MS traffic, mapped as BE flow, has the lowest priority. When MS is connected to BS1, resources are firstly granted to SS1 UGS connection, and the remaining resources are assigned to the MS BE connection. The same happens after the first handover, where BS2 serves SS2 rtPS traffic through a request mechanism, penalizing MS traffic flow. However, BS3 shares its network resources between SS3 and MS in a best effort contention after the second MS handover.

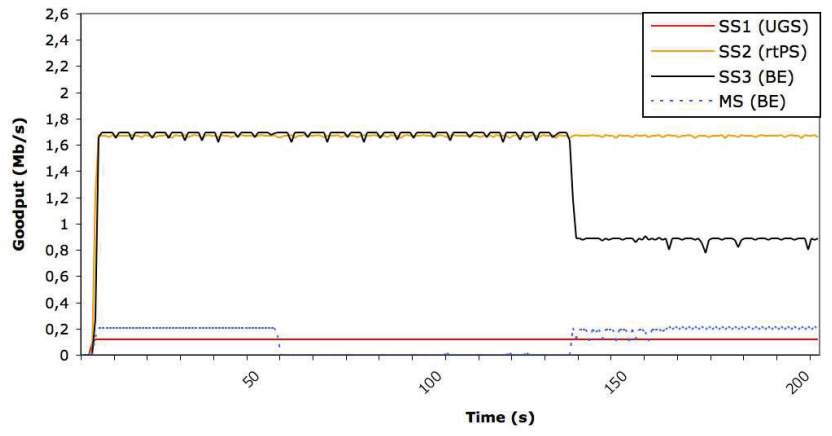

Figure 16. BE goodput during mobility (SIM3e).

It should be highlighted that, when MS performs a handover from a serving $\mathrm{BS}$ to a target $\mathrm{BS}$, unused network resources are released after the Resource Retain Timer expiration. Hence, despite the NIST module implementation in [3], in the proposed module these resources are reassigned to SSs inside the old serving BS, avoiding bandwidth wasting.

\section{CONCLUSIONS}

In this paper we have described the development of a new simulation module for NS2, based on IEEE 802.16 standard, designed to support QoS management during mobility in WiMAX environments.

The functionalities offered by the realized NS2 module have been validated through extensive simulations and its standardcompliant QoS resource allocation has been tested. The module provides mobility support within WiMAX networks, showing how QoS can be managed and maintained during mobility.

We believe that this effort could constitute a significant contribution to the research community involved in resource allocation and QoS issues over fixed and mobile WiMAX networks.

\section{REFERENCES}

[1] IEEE Standard for Local and Metropolitan Area Networks Part 16: Part 16: Air Interface for Broadband Wireless Access Systems. IEEE Std., 2009.

[2] The network simulator ns-2. http://www.isi.edu/nsnam/ns/, October 2010

[3] NIST, "The Network Simulator NS-2 NIST Add-on IEEE 802.16 Model (MAC+PHY)". http://www.nist.gov/itl/antd/emntg/ssm_tools.cfm, October 2010.

[4] Belghith, A., and Nuaymi, L., Design and Implementation of a QoS-included WiMAX Module for NS-2 Simulator. In Proceedings of the International Conference on Simulation Tools and Techniques for Communications, Networks and Systems \& Workshops (Marseille, France, March 3-7, 2008). SIMUTOOLS '08.

[5] Fonseca, N.L.S., and Freitag, J., WiMAX Module for the NS-2 Simulator. In Proceedings of the $18^{\text {th }}$ International Symposium on Personal, Indoor and Mobile Radio Communications (Athens, Greece, September 3-7, 2007) PIMRC '07, 1-6.

[6] Deploying license-exempt WiMAX solutions. Technical report, Intel, 2005.

[7] Considerations for deploying mobile WiMAX at various frequencies. Technical report, Nortel, 2006.

[8] Mobile WiMAX - Part I: A Technical Overview and Performance Evaluation. WiMAX Forum, 2006.

[9] Belghith, A., and Nuaymi, L., Comparison of WiMAX scheduling algorithms and proposal for the rtPS QoS class. In Proceedings of the $14^{\text {th }}$ European Wireless Conference (Prague, Czech Republic, June 22-25, 2008). EW '08, 1-6.

[10] Shreedhar, M., and Varghese, G., Efficient fair queuing using Deficit Round-Robin, ACM/IEEE Transactions On Networking, 4 (3), 375-385, June 1996. 